\title{
Estimation of the influence of finite dielectric substrates on the far-field pattern of an array of metallic scatterers in the infrared
}

\author{
José María Rico-García, José Manuel López-Alonso, Javier Alda* \\ Optics Department, University Complutense of Madrid, School of Optics, Av. Arcos de Jalón s/n. 28037 Madrid, Spain
}

Received 14 November 2003

\begin{abstract}
The far-field scattered in the infrared by an arrangement of metallic structures deposited on a dielectric wafer is estimated in this paper. The scattering is modelled by using operators that describe the far field obtained under the regime applicable for the Babinet's principle in its vectorial approach and the Stratton-Chu approximation. The farfield scattered by an arrangement of thin gold layers over a dielectric wafer under infrared illumination is computed. The model assumes a normally incident vectorial Gaussian beam focused over the arrangement plane. An angular spectrum decomposition of the field is done. Then, every plane wave is scattered by the whole structure: arrangement + substrate layer. The reflexions in the substrate layer and the arrangement action have been taken into account in an operator formalism. Numerical results estimating the influence of substrate thickness on the pattern are obtained.

(C) 2004 Published by Elsevier B.V.
\end{abstract}

\section{Introduction}

Novel devices and concepts, as frequency selective surfaces or antenna-coupled detectors in the infrared, are being developed by writing metallic structures with spatial dimensions in the wavelength range, on dielectric wafers [1-4]. For the infrared, $\mathrm{Si}$ wafers are intensively used because

\footnotetext{
${ }^{*}$ Corresponding author. Tel.: +34-91-394-6874; fax: +34-91394-6885.

E-mail addresses: jmrico@fis.ucm.es (J.M. Rico-García), jmlopez@opt.ucm.es (J.M. López-Alonso), j.alda@fis.ucm.es (J. Alda).
}

of its availability and transparency above $\lambda=$ $9.7 \mu \mathrm{m}$. These Si wafers may be two-sides polished allowing a substrate-side incidence [5]. At the same time, the presence of two plane surfaces with optical quality on both sides of the wafer makes possible to observe interference effects. Therefore, the back surface (we name front surface to that one containing the metallic structures, the back surface is the other one) is playing a role that should be considered more in detail. The analysis of frequency selective surfaces made by numerical method has been focused in the computation of the spectral changes produced by the metallic structures [6]. In order to gain insight on the relation between geometry and selective 
behaviour, some other parameters or effects have been neglected. One of these effect is the influence of the substrate thickness on the radiation reflected by the structure. Codreanu and Boreman [7] analyze the effect of the dielectric substrate and the ground plane location for microstrip antennas. In this paper we show a method to specifically analyze the effect of the substrate thickness on the spatial-frequency spectrum scattered by a metallic structure.

The size of the metallic structures analyzed in this paper is of the order of the wavelength. This is the reason why we will use the term scattering, or scatterers, to refer the effect of the interaction of light with these metallic structures in order to find the far-field pattern. Section 2 is devoted to a detailed explanation of the proposed method where the successive reflections and scatterings are described by an operator formalism. In Section 3 we obtain the form of the involved operators. Our approach is based on the Babinet principle applied in its vectorial form [8]. The Stratton-Chu approximation will be also used to analytically evaluate the scattered field when inciding with actual vectorial beams on wavelength-sized metallic structures [9]. The necessary conditions to apply these approximations are discussed before applying it to actual structures in Section 4. In Section 4 we have obtained the far-field pattern for a typical case of interest where an arrangement of five metallic squares is illuminated by an infrared Gaussian beam. The incoming beam is also modelled in this section. After checking the fulfilment of the necessary hypothesis we apply the formalism to a simple case as an example. The far-field patterns have been obtained by numerical calculation using the method presented in this paper. The dependences of these patterns with the thickness of the wafer, and with the spatial content of the incoming beam, are analyzed. Finally, Section 5 summarizes the main conclusions and results of this paper.

\section{Operator method to model the effect of the finite substrate}

The description of the successive scattering processes produced by microlithographic elements written on a finite-thickness wafer is made in this paper by using an operator formalism. The main idea is to establish a correspondence between a single scattering or reflection process with a mathematical operator applied to the incident field. Then, the complicated interaction between the beam and the structure can be decomposed into easier problems. By taking only the most relevant ones, it is possible to obtain a useful insight of the whole problem.

Fig. 1 shows an arrangement of metallic scatterers on a finite dielectric substrate. A beam impinging on the structure is reflected and scattered by it. When considering the successives reflection and scattering events, the $\vec{E}$ field observed on a plane located above the arrangement can be expressed as:

$\vec{E}=\vec{E}_{0}+\vec{E}_{\mathrm{s}}$,

where $\vec{E}_{0}$ is the unperturbed field, i.e., the field in absence of any arrangement. It can be obtained from the incident beam. $\vec{E}_{\mathrm{s}}$ is the scattered field above the arrangement plane. The total field on that plane is the sum of both. As we can see from Fig. $1, \vec{E}_{\mathrm{s}}$ is the sum of successive scattering and reflection processes:

$\vec{E}_{\mathrm{s}}=\vec{E}_{\mathrm{s}}^{(1)}+\vec{E}_{\mathrm{s}}^{(2)}+\vec{E}_{\mathrm{s}}^{(3)}+\cdots$

We can represent the scattering action of the arrangement on the electric field as an operator, $\mathbf{K}_{1,2}$, where the subindices 1,2 mean that the light travels from medium 1 to medium 2 . This operator

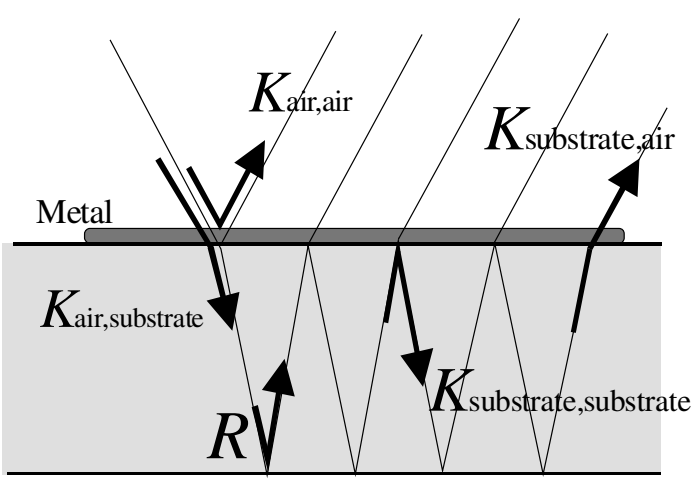

Fig. 1. Graphical scheme for the definition of the $\mathbf{K}_{1,2}$ and $\mathbf{R}$ operators. 
applies to the electric field inciding on the arrangement and produces the reflected or transmitted scattered field. In the same way, the reflection by the back side of the substrate (see Fig. 1) can be also described by an operator, $\mathbf{R}$, applied to the incident field and producing the reflected field by the back side interface.

By using these operators it is possible to compute the contributions to $\vec{E}_{\mathrm{s}}$. For example, the first term is given as:

$\vec{E}_{\mathrm{s}}^{(1)}=\mathbf{K}_{\text {air }, \text { air }}\left(\vec{E}_{0}\right)$,

where the subindex air,air denotes where the light is coming from and where the scattered light is propagating to. The second term contribution reads as:

$\vec{E}_{\mathrm{s}}^{(2)}=\mathbf{K}_{\text {substrate,air }} \mathbf{R} \mathbf{K}_{\text {air,substrate }}\left(\vec{E}_{0}\right)$.

Therefore, the $p$ th contribution to the scattered field above the structure, for $p>1$, is:

$\vec{E}_{\mathrm{s}}^{(p)}=\mathbf{K}_{\text {substrate, air }}\left(\mathbf{R K}_{\text {substrate,substrate }}\right)^{p-2} \mathbf{R} \mathbf{K}_{\text {air,substrate }}\left(\vec{E}_{0}\right)$.

All these contribution can be summarized to obtain the total field above the scatterers plane as follows:

$\vec{E}=\mathbf{U}^{-}\left(\vec{E}_{0}\right)$,

where the operator $\hat{\mathbf{U}}^{-}$is defined by the following equation:

$$
\begin{aligned}
\mathbf{U}^{-}= & \mathbf{1}+\mathbf{K}_{\text {air,air }}+\sum_{p=2}^{\infty} \mathbf{K}_{\text {substrate,air }} \\
& \times\left(\mathbf{R K}_{\text {substrate,substrate }}\right)^{p-2} \mathbf{R} \mathbf{K}_{\text {air,substrate }}
\end{aligned}
$$

The influence of the finite substrate in the scattered field is included in $\mathbf{R}$ operator and $\vec{E}_{0}$ field, which is computed previously and without the scatterers array by using the incident beam, and taking into account the presence of the finite substrate.

\section{Construction of the $K$ and $R$ operators}

In order to obtain the scattered field we first need to know the actual form of the operators involved in the calculations. We will base their construction on some physical principles properly justified. We have used a vectorial diffraction integral to precise the mathematical form of the $\mathbf{K}_{1,2}$ operator [8]. To define the reflection operator, we carry out a plane-wave decomposition of the incident electric field [10]. Then, each plane-wave component is reflected. This approach produces an equation relating the incident field and the reflected one: the $\mathbf{R}$ operator.

\subsection{The $\mathbf{K}$ operator}

When the depth of penetration is much smaller than the thickness of the metal layer, we may assume that the metal behaves as a perfect electric conductor. This approximation simplifies the analysis made in the following sections and it will be properly justified. Moreover, when the metal thickness is far less than the wavelength of the electromagnetic radiation, then the metal structure can be taken as a thin layer with zero thickness. Under these approximations, the far-field scattered by an array of metallic structures deposited on a substrate can be computed making use of the vectorial diffraction integral and the Babinet principle [8].

The calculation begins applying the Babinet principle to the original problem, solving the complementary problem, and retrieving the initial one. The Babinet principle relates the electric and magnetic fields in the complementary (denoted with a c subscript) and original problems as follows [8]:

$\vec{E}=\vec{E}_{\text {incident }}+v \vec{B}_{\mathrm{c}}$,

where $v$ is the speed of light in the medium. On the other hand electric and magnetic fields obey the transversality condition in the far field:

$\vec{B}_{\mathrm{c}}=\frac{\mu_{0}}{Z}\left(\vec{u} \times \vec{E}_{\mathrm{c}}\right)$,

where $Z$ is the impedance of the medium, and $\vec{u}$ is an unitary vector along the direction of propagation. Finally, to calculate the solution in the far-field range we apply the far-field form of the Green's propagator into the diffraction integral [8], 


$$
\begin{aligned}
\vec{E}_{\mathrm{c}}= & \pm\left(\frac{\mathrm{i} \mathrm{e}^{\mathrm{i} k r}}{2 \pi r} k v\right) \vec{u} \times \int_{\text {aperture }} \vec{n} \\
& \left.\times \vec{B}_{\text {incident }} \mathrm{e}^{-\mathrm{i}\left(k_{x} x+k_{y} y\right.}\right) \mathrm{d} x \mathrm{~d} y,
\end{aligned}
$$

where $\vec{n}$ is an unitary vector perpendicular to the interface, and $\vec{k}=\left(k_{x}, k_{y}, k_{z}\right)$ is the wave vector in the medium. The \pm denotes the forward or backward propagation. Introducing Eq. (10) into Eq. (8) and making use at the same time of Eq. (9), we obtain

$$
\begin{aligned}
\vec{E}_{\text {scattered }}= & C \frac{\mathrm{e}^{\mathrm{i} k_{2} r}}{r} \vec{u} \times \vec{u} \times \iint_{\text {aperture }} \vec{n} \\
& \times \vec{B}_{\text {incident }} \mathrm{e}^{-\mathrm{i}\left(k_{1 x} x+k_{1 y} y\right)} \mathrm{d} x \mathrm{~d} y \\
= & \mathbf{K}_{1,2}\left(\vec{E}_{\text {incident }}\right),
\end{aligned}
$$

where $k_{1}$ and $k_{2}$ are the wavenumbers of the radiation in the media where the incident field and the scattered field are propagating, respectively. In this last equation $r$ is the distance from the observation point to the coordinate origin, placed in the center of the complementary aperture, $r=\sqrt{x^{2}+y^{2}+z^{2}}$ (we assume that the integration is made on the $z=0$ plane), $\vec{u}$ is a unitary vector pointing out in the same direction as $\vec{r}$, and $\vec{B}_{\text {incident }}$ is the magnetic field associated to $\vec{E}_{\text {incident }}$. Finally, $C$ is a constant defined as

$$
C= \pm \frac{\mathrm{i} v^{2} \mu_{0}}{Z \lambda}
$$

where $\mu_{0}$ is the magnetic permeability in vacuum, and $\lambda$ is the wavelength in the medium where the scattered wave is travelling to. This last equation allows to define a scattering amplitude as follows:

$\vec{F}=\vec{u} \times \vec{u} \times \iint_{\text {aperture }} \vec{n} \times \vec{B}_{\text {incident }} \mathrm{e}^{-\mathrm{i}\left(k_{1 x} x+k_{1 y} y\right)} \mathrm{d} x \mathrm{~d} y$.

The squared modulus of this function will be represented in the last section of this paper to describe the effect of the operators defined here.

In this section, the most stringent assumption that we have employed to obtain $\vec{E}_{\text {scattered }}$ is that the total tangential electric field in the plane of the complementary aperture is replaced by the tangential component of the incident electric field: $\vec{n} \times \vec{E}_{\text {total }} \simeq \vec{n} \times \vec{E}_{\text {incident }}$.

In the complementary aperture $\vec{n}$ is the normal to the aperture and the term $\vec{n} \times \vec{E}$ represent an equivalent current. This approximation means that the total equivalent electric current in the aperture can be replaced by the equivalent current induced by the incident field. This is known as the Stratton-Chu approximation. The relation fails if the lateral dimension of the aperture is close to the wavelength. However, for rectangular apertures, the Stratton-Chu approximation is correct even for dimensions of the order of the wavelength [9], and this is the case we are interested in.

\subsection{The $\mathbf{R}$ operator}

The $\mathbf{R}$ operator applies to the incident field on the interface between two media of different index and produces the reflected field. The geometry of the problem is shown in Fig. 2. The plane-wave angular spectrum of the reflected beam is obtained after applying the reflection coefficients [11] to each one of the plane-wave components represented by its plane-wave spectrum, $\vec{A}_{\text {inc }}$. However, to properly reflect each one of them, we have to move from the original reference system to a "local" one, (see Ref. [12], for example) by means of a

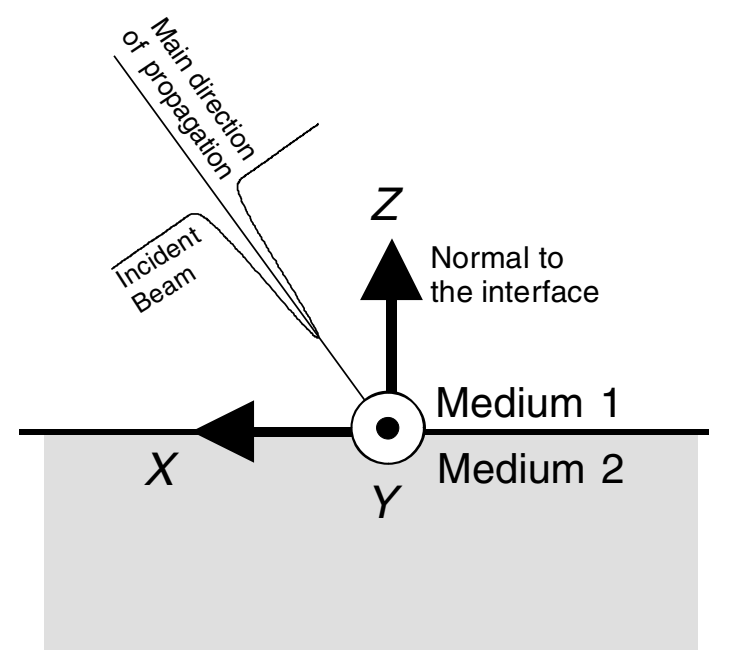

Fig. 2. Location of the plane of incidence defined for the chief ray of the incoming beam. 


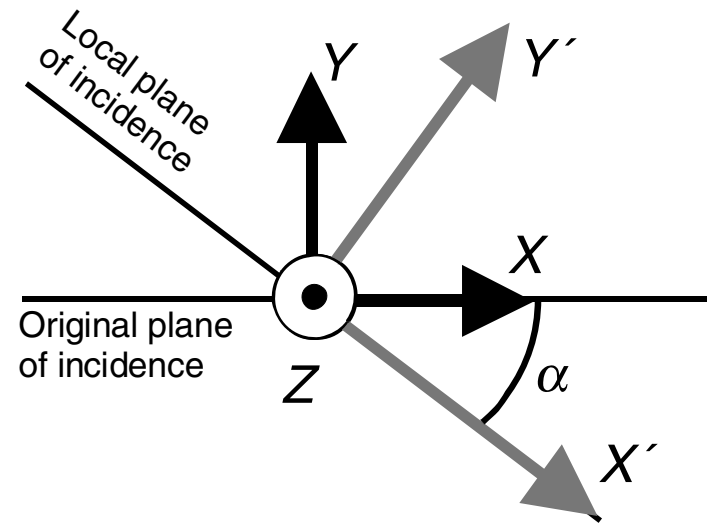

Fig. 3. Location of the "local" plane of incidence defined for an arbitrary plane wave component of the incoming beam. The reference system is rotated around the $Z$ axis by an angle $\alpha$ to become the "local" reference system.

rotation (see Fig. 3). Within the "local" reference system, the incident and reflected field are connected by the following reflection matrix,

$\hat{\rho}_{\text {local }}=\left(\begin{array}{ccc}r_{\perp} & 0 & 0 \\ 0 & r_{\|} & 0 \\ 0 & 0 & r_{\|}\end{array}\right)$,

where $r_{\perp}$ is the reflection coefficient for perpendicular polarization and $r_{\|}$is the reflection coefficient for parallel polarization. By moving again to the original reference system, $\hat{\rho}$ becomes

$\hat{\rho}=\left(\begin{array}{ccc}r_{\perp} \cos ^{2}(\alpha)+r_{\|} \sin ^{2}(\alpha) & \left(r_{\perp}-r_{\|}\right) \sin (\alpha) \cos (\alpha) & 0 \\ \left(r_{\perp}-r_{\|}\right) \sin (\alpha) \cos (\alpha) & r_{\perp} \cos ^{2}(\alpha)+r_{\|} \sin ^{2}(\alpha) & 0 \\ 0 & 0 & r_{\|}\end{array}\right)$,

where $\alpha$ is the angle formed by the $X Z$ plane and the "local" incidence plane of each plane-wave of the field.

After reflecting all the plane waves in the beam, we propagate them towards an observation plane of $z=$ constant, and add them to find the total reflected electric field in this plane:

$$
\begin{aligned}
\vec{E}_{\text {ref }} & =\iint \hat{\rho} \cdot \vec{A}_{\text {inc }} \mathrm{e}^{-\mathrm{i} k_{z 1} z} \mathrm{e}^{\mathrm{i}\left(k_{1 x} x+k_{1 y} y\right)} \mathrm{d} k_{x} \mathrm{~d} k_{y} \\
& =\hat{\mathbf{R}}\left(\vec{E}_{\text {inc }}\right)
\end{aligned}
$$

where the $\mathbf{R}$ operator is defined.

\section{Application of the method to a practical case}

In this section we have computed the far-field behaviour of the scattered field in a $z$ plane when a monochromatic Gaussian beam interacts with an arrangement of metallic scatterers located on the surface of a finite substrate. This arrangement is modelled as an example to prove the feasibility of the method. Larger structures with some other geometries and symmetries can also be analyzed by using the operator model described above.

To properly analyze this example we need a vectorial model for the beam inciding onto the array plane. A good way to manage with this problem is to obtain the angular spectrum of the field, and define it in the Fourier space. Once we have done this, evaluating the scattered field changes to an easier task if we truncate the Eq. (7) to a given order. Even by taking into account the lowest order reflection and scattering processes we may distinguish the effect of the finiteness of the substrate on the far-field pattern.

\subsection{Fourier-space characterization of the beam}

The scatterers are illuminated by a laser Gaussian beam, focused on the arrangement plane, and inciding normally. If we assume that the beam is linearly polarized with its field along the $X$ direction, the component of the electric field along this direction is written as

$E_{x}=E_{x 0} \exp \left[-\left(\frac{x^{2}+y^{2}}{\omega_{0}^{2}}\right)\right]$,

where $\omega_{0}$ is the beam waist at the focus of the beam, and $E_{x 0}$ is the maximum amplitude at the beam waist. A monochromatic vectorial electric field in a homogeneous medium must satisfy the Helmholtz equation

$\vec{\nabla}^{2} \vec{E}+k^{2} \vec{E}=0$.

The solutions for this equation can be expanded as a superposition of plane waves as follows [10]

$\vec{E}(x, y ; z)=\iint \vec{A}(\xi, \eta ; z) \mathrm{e}^{(\overrightarrow{\mathrm{i}}(\xi, \eta) \vec{r})} \mathrm{d} \xi \mathrm{d} \eta$,

where $\vec{r}=(x, y, z)$, and $\xi, \eta$ are the spatial frequencies associated with the plane-wave spectrum, 
$\vec{A}(\xi, \eta ; z)$. This spectrum can be understood as the complex vector amplitude associated to the planewave travelling in the $\vec{k}$ direction, being $\vec{k}$ defined by

$\vec{k}=\frac{2 \pi}{\lambda}\left(\lambda \xi, \lambda \eta, \sqrt{1-\lambda^{2}\left(\xi^{2}+\eta^{2}\right)}\right)$,

where $\lambda$ is the wavelength inside the medium. The evanescent waves occur when $\left(\xi^{2}+\eta^{2}\right)>\left(1 / \lambda^{2}\right)$. We will neglect the contribution of these evanescent waves in the conditions for the applicability of the method.

By using the Gauss's equation for the electric field, $\vec{\nabla} \cdot \vec{E}=0$, it is possible to obtain the following relation between the direction of propagation of the plane waves and the amplitude of the plane-wave spectrum,

$\vec{k}(\xi, \eta) \cdot \vec{A}(\xi, \eta ; z)=0$.

This condition means that all the plane waves must be transversal to its wave vector. This equation establishes a constrain between the three components of the $\vec{E}$ field. In our example, and without lack of generality, we will set the value of the $E_{y}$ component equal to zero (TM beam). Then, after applying Eq. (22), we obtain the $z$ component of the plane wave spectrum as

$A_{z}(\xi, \eta)=-\frac{k_{x}}{k_{z}} A_{x}(\xi, \eta)$

being the beam completely defined in Fourier space. In Fig. 4 we have represented them squared modulus of the two non-null components of a Gaussian beam having $E_{y}=0$ having a Gaussian width of $10 \mu \mathrm{m}$ and a wavelength of $10 \mu \mathrm{m}$. The direction of propagation is $Z$. Please note that when normalizing the $E_{x}$ component to 1, the squared modulus of the $E_{z}$ component reaches a maximum value of only $2.2 \%$ of the maximum value of the squared modulus of the $E_{x}$ component.

\subsection{Approximations to the field}

The basic unit of the array of scatterers analyzed in this paper, as an example of the applicability of the method, is a squared thin gold layer with dimension $10 \times 10 \mu \mathrm{m}$. The gold layer is 200 $\mathrm{nm}$ thick. The arrangement consists of five of these basic units located in a star configuration where the center of the scatterers are $20 \mu \mathrm{m}$ apart along the $X$ and $Y$ directions. The substrate is made of silicon and corresponds with a two-sides-polished wafer. The thickness of the wafer is $300 \mu \mathrm{m}$. The illumination of the array is done with a $\mathrm{CO}_{2}$ laser emitting at $10 \mu \mathrm{m}$. The electric conductivity [13] at these optical frequencies is $\sigma=2.6 \times 10^{6} \Omega^{-1} \mathrm{~m}^{-1}$. Then, the penetration depth is obtained as

$\delta \simeq \sqrt{\frac{2}{\mu \sigma(\omega) \omega}}$.

The silicon substrate is transparent in the infrared wavelength used in this paper. Therefore, the index of refraction [14] is a real number having a value of $n=3.42$. By using the previous conditions it is possible to evaluate the penetration thickness for the optical frequencies of a $\mathrm{CO}_{2}$ laser emission at $10 \mu \mathrm{m}$. This penetration depth is $\delta \simeq 0.06 \mu \mathrm{m}$. This value is much lesser than the thickness of the metallic structure that is about 0.2 $\mu \mathrm{m}$. This means that, for the purpose of this paper, the metal can be taken as a perfect electric conductor. On the other hand, the thickness of the metallic structure is much lesser than the wavelength of the optical radiation. Then, the scatterer can be seen as a planar structure. Summarizing, we are dealing with a thin, planar, perfect electric conductor and the conditions under which the $\mathbf{K}$ and $\mathbf{R}$ operator are developed are fulfiled.

In the previous calculation we have found the structure of the operators involved in Eq. (7). An easy way of evaluating the field is to truncate the series until a given order. For example, the first and second order approximations have the following form,

$\vec{E}=\left(\mathbf{1}+\mathbf{K}_{\text {air,air }}\right)\left(\vec{E}_{0}\right)$,

$\vec{E}=\left(\mathbf{1}+\mathbf{K}_{\text {air,air }}+\mathbf{K}_{\text {substrate,air }} \mathbf{R} \mathbf{K}_{\text {air,substrate }}\right)\left(\vec{E}_{0}\right)$,

where $\vec{E}_{0}$ is the unperturbed field, i.e., the field existing without any scattering structure. To begin the application of these operators we need the exact unperturbed tangential field on the plane of 


\section{ARTICLE IN PRESS}

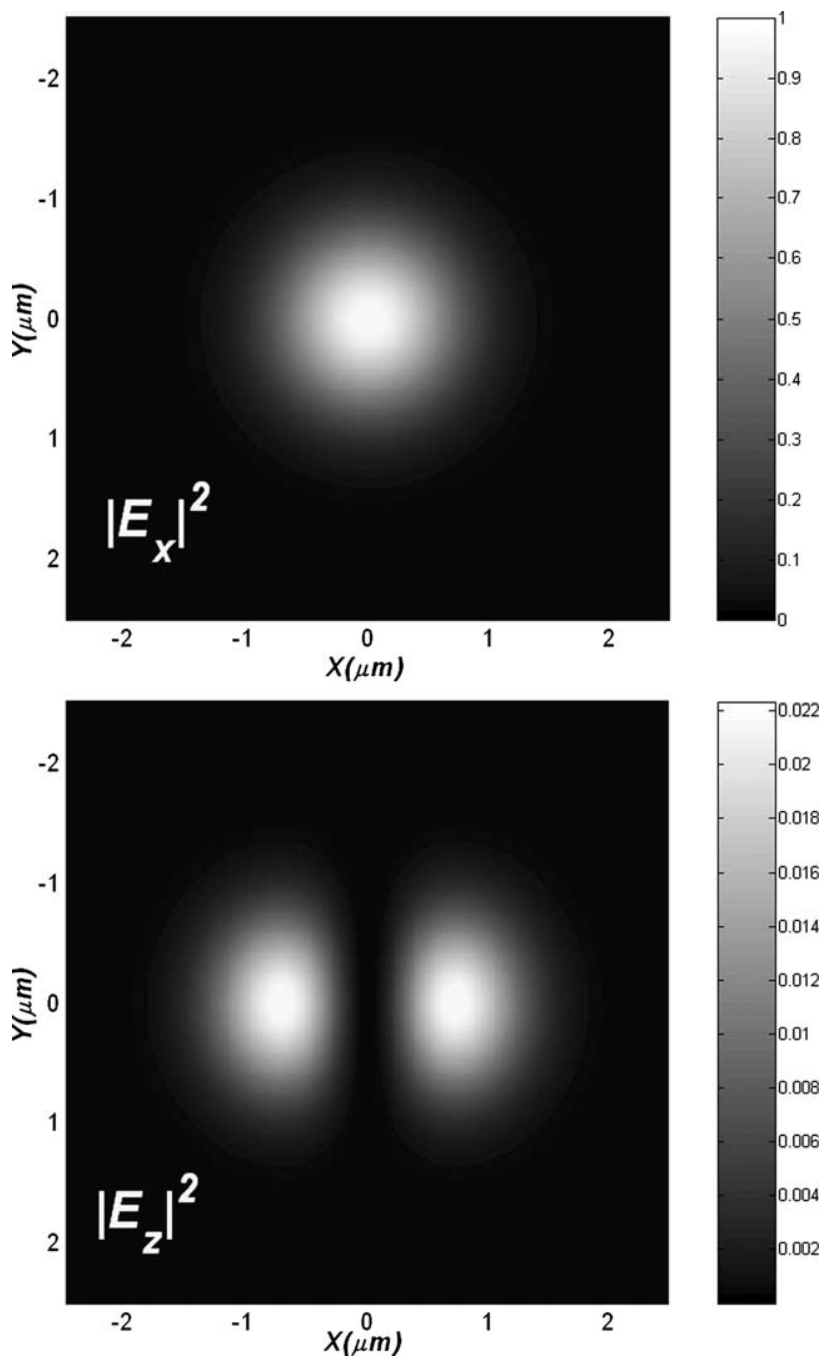

Fig. 4. Field distributions of the two non-null components of the incident Gaussian beam used in this paper.

the scatterers. When inciding with the previously modelled beam the exact tangential field is the sum of the incident tangential field and the reflected tangential field. This can be expressed as follows,

$\vec{E}_{0, \mathrm{~T}}=\vec{E}_{\mathrm{inc}, \mathrm{T}}+\vec{E}_{\mathrm{ref}, \mathrm{T}}$

and

$\vec{E}_{\text {ref }}=\hat{\mathbf{R}}\left(\vec{E}_{\text {inc }}\right)$.

The reflection operator for the layer, $\hat{\mathbf{R}}$, has the same form as the reflection operator for an interface, except for the reflection coefficients [11], that in this last case takes into account the existence of the successive reflections on the wafer. Therefore, the influence of the substrate appears in the first order contribution already, because of the presence of the $\vec{E}_{0}$ field.

\subsection{Results}

In the following calculation we have restricted our analysis to the first term of the series (Eq. (25)), which represents the most important contribution to the scattered field by the array. We 
compute the scattering amplitude, $\vec{F}$, of $\vec{E}_{\text {s }}$ field to first order (see Eq. (13)). This approach makes sense because the scattered field has the following form:

$\vec{E}_{\mathrm{s}}=C \frac{\mathrm{e}^{\mathrm{i} k r}}{r} \vec{F}$

This corresponds with the electric field at the far zone. The scattering amplitude, $\vec{F}$, is a complex vector function containing all the information about how the energy is being scattered by the arrangement. This scattering amplitude can be derived after applying the $\mathbf{U}-\mathbf{1}$ operator to the $\vec{E}_{0}$ field. In this paper we have truncated the operator series until first order. Figs. 5-7 represent the $|\vec{F}|^{2}$ value at $z=300 \mu \mathrm{m}$ from the plane where are placed the scatterers. This distance is far enough to
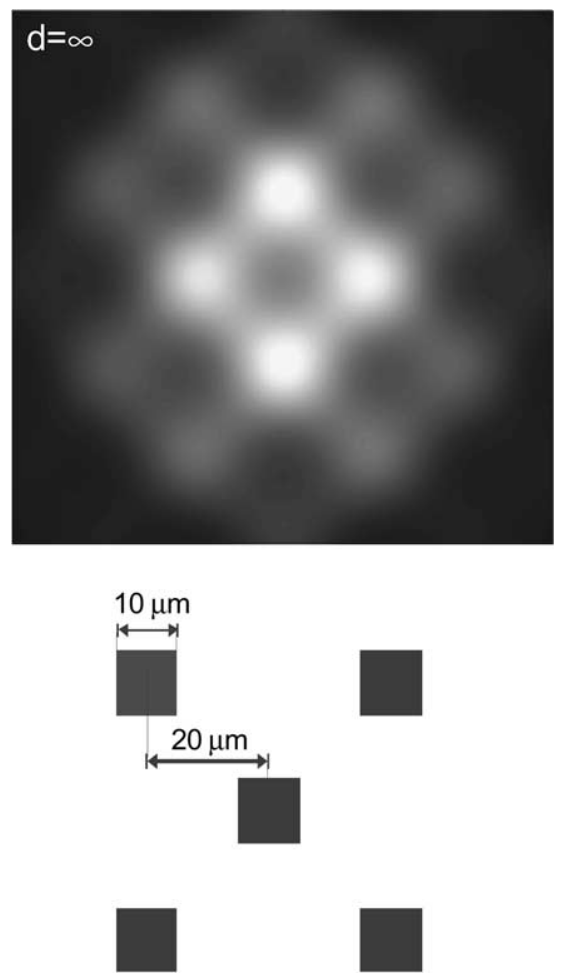

Fig. 5. Squared modulus of the scattering amplitude when a Si wafer presents infinite thickness. The dimensions and arrangement of the scatterers is represented in the bottom of this figure. The incoming field belongs to a Gaussian beam, having a beam waist width, $\omega_{0}=2 \lambda$, being $\lambda=10 \mu \mathrm{m}$. The observation plane is located $300 \mu \mathrm{m}$ above the scatterers plane.
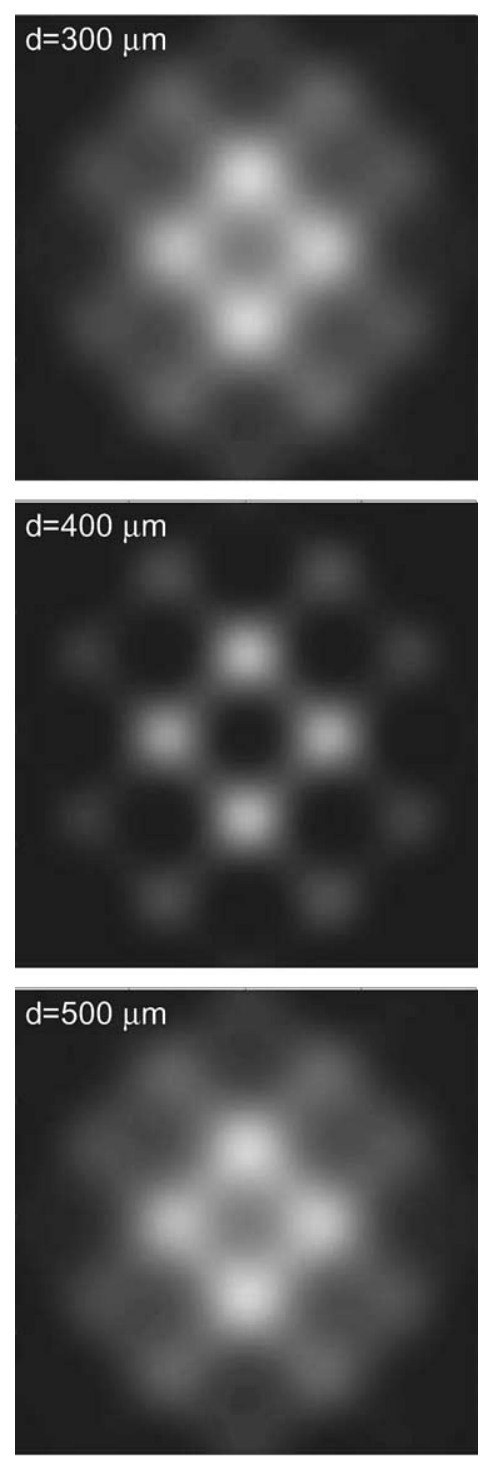

Fig. 6. Squared modulus of the scattering amplitude, $|\vec{F}|^{2}$, for $d=300,400$ and $500 \mu \mathrm{m}$. The incidence and observation conditions are the same than in Fig. 5.

use the far-field approximation. This approximation is fully complied if the following condition is fulfiled [10]:

$z \gg \frac{k l^{2}}{2}$

where $l$ is the characteristic size of the scatterer. In our case, after substituting the values used in 

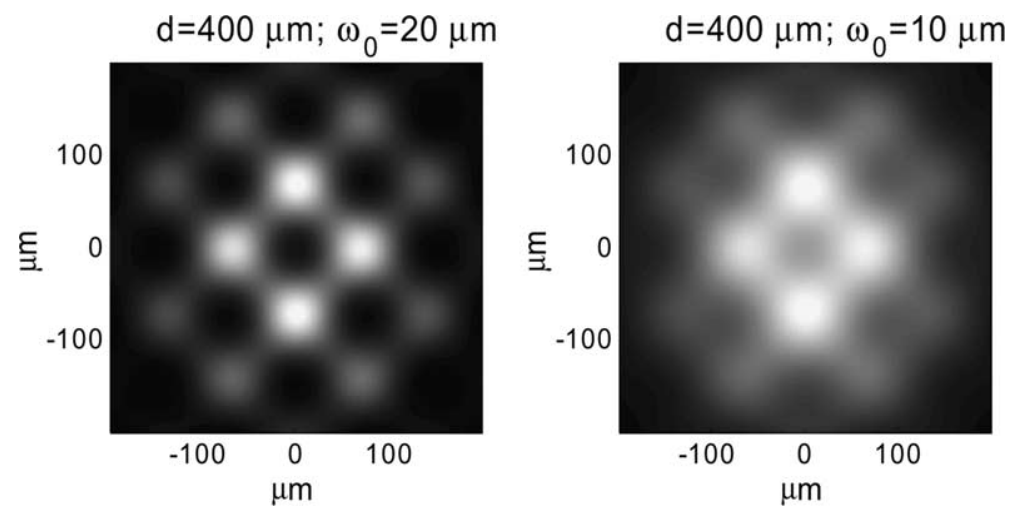

Fig. 7. Comparison between different incidence conditions: the two figures shows the far-field pattern at the same plane. The picture on the left is $|\vec{F}|^{2}$ when the beam waist, $\omega_{0}$, is two times the wavelength whereas in the picture on the right the waist is equal to the wavelength. The maxima and minima are reinforced or weakened depending on the spatial spectral content of the beam, i.e., the number of plane waves whom is composed.

this study, the checking of this condition is positive.

In Fig. 5 we have represented the squared modulus of the amplitude of scattering for the scatterers structure shown at the bottom of this figure and in the condition of semi-infinity substrate. The series is truncated and only the first $\mathbf{K}_{\text {air,air }}$ operator applies. Fig. 6 shows $|\vec{F}|^{2}$ for three finite substrate cases. The substrate thickness, $d$, takes three values: 300,400 , and $500 \mu \mathrm{m}$. The incident beam has been modelled in the previous subsection. In these figures we can see the dependence of maxima and minima of the far-field pattern with the wafer thickness. This dependence is due to the interference phenomena related to a Fabry-Perot behaviour associated with the thickness of the wafer. By adopting an angular spectrum point of view the understanding of this behaviour is easier. The incident field is a linear combination of plane-waves travelling in various directions. Each one is affected by scattering processes in the arrangement and reflection processes in the back side of the wafer. Changing the wafer thickness implies the modification of the reflection processes, because the reflection coefficients of the layer are altered (the optical path inside the wafer is different changing the phase). On the other hand, by varying the thickness of the wafer, the portion of the spatial-frequency spectrum arriving again to the arrangement of scatterers will also change, modifying the far-field pattern. Therefore, the far-field pattern will show a dependence on the wafer thickness, even since the first order approach. In the analyzed figures (see Fig. 6 where the gray levels are the same for the three figures) we have plotted patterns showing different contrasts between maxima and minima. However, the locations of maxima and minima and general structure of the pattern are not strongly affected by changes in the thickness of the wafer. This general structure is also preserved for the case of infinity thickness (Fig. 5), and it is primarily related with the geometry of the arrangement of scatterers.

Another noticeable dependence is the variability of the pattern with the beam spatial spectral content for a constant thickness. In Fig. 7 we have represented the variation in $|\vec{F}|^{2}$ with respect to the spatial-frequency content of the beam, that depends on the beam waist width, $\omega_{0}$. The reason for this difference is that the spectral content is driven by the value of the waist: the bigger the waist, the narrower the angular spectrum of the beam. If the content of plane waves are not the same, it means there are some plane waves with significative amplitude in one of the beam which are not present in the other. Therefore, the interference between the scattered waves must be different from one pattern to another. 


\section{Conclusions}

We have studied the influence of the wafer thickness in the field scattered by an arrangement of thin metal layers deposited on the wafer. The approach developed here is based on an operator formalism, properly designed under reasonable hypothesis. The method allows the quantitative estimation of the importance of substrate thickness on the spatial structure of the scattered radiation in the far field. The hypothesis about the characteristics of the metallic structures and their fulfilment has been specifically analyzed and checked for the case treated here. The example corresponds with the case of metallic structures written with e-beam lithography and used for a variety of applications in the infrared (frequency selective surface, antenna-coupled detectors, etc.). The main effect we have modelled is the dependence of the scattering pattern with the wafer thickness. We have calculated these patterns using the previously mentioned operator approach until the first order approach. Successive terms of the series can be taken to refine the results and study in more detail the scattering pattern. From the obtained results we have checked that the maxima and minima of the $|\vec{F}|^{2}$ on a constant $z$ plane can be enhanced or reduced depending on the wafer thickness. However, the general structure of the pattern is related with the geometry of the scatterers and their interaction with the incident beam. Therefore, this general structure is not strongly affected by the wafer thickness. Dependence with the spatial spectral content of the field has been also analyzed. From a comparison between the infinite thickness case and the rest it can be concluded that the finite layer is responsible of interference effects in the far-field pattern that modifies the contrast of the irradiance pattern in the far field remaining the structure basically unchanged. This result justifies some previous findings of other authors where the wafer thickness effect is neglected $[2,6]$. On the other hand, the evaluation of the far-field distribution may be used to obtain, by a balance equation, the energy actually absorbed by the metallic structure. This could be of particular importance when evaluating the energy coupled to optical antennas structures.

\section{Acknowledgements}

This work has been supported by the Ministerio de Ciencia y Tecnología of Spain, under the project TIC2001-1259.

\section{References}

[1] S.E. Schwarz, B.T. Ulrich, Antenna coupled infrared detectors, J. Appl. Phys. 48 (1977) 1870-1873.

[2] I. Puscasu, W.L. Schaich, G.D. Boreman, Modeling parameters for the spectral behavior of infrared frequency-selective surfaces, Appl. Opt. 40 (2001) 118-124.

[3] C. Fumeaux, J. Alda, G. Boreman, Lithographic antennas at visible frequencies, Opt. Lett. 24 (1999) 1629-1631.

[4] K.B. Crozier, A. Sundaramurthy, G.S. Kino, C.F. Quate, Optical antennas: resonators for local field enhancement, J. Appl. Phys. 94 (2003) 4632-4642.

[5] J. Alda, C. Fumeaux, M.A. Gritz, D. Spencer, G. Boreman, Responsivity of infrared antenna-coupled micro-bolometers for air-side and substrate-side illumination, Infr. Phys. Technol. 41 (2000) 1-9.

[6] W.L. Schaich, G. Schider, J.R. Krenn, A. Leitner, F.R. Aussenegg, I. Puscasu, B. Monacelli, G. Boreman, Optical resonances in periodic surface arrays of metallic patches, Appl. Opt. 42 (2003) 5714-5721.

[7] I. Codreanu, G. Boreman, Influence of dielectric substrate on the responsivity of microstrip dipole-antenna-coupled infrared microbolometers, Appl. Opt. 41 (2002) 1835-1840.

[8] J. Jackson, Classical Electrodynamics, third ed., Wiley and Sons, New York, 1998.

[9] J. Stratton, L. Chu, Diffraction theory of electromagnetic waves, Phys. Rev. 56 (1939) 99-107.

[10] J.W. Goodman, Introduction to Fourier Optics, McGrawHill, New York, 1968.

[11] L. Pedrotti, F. Pedrotti, Optics, Prentice-Hall International, New Jersey, 1996.

[12] J. Alda, Transverse angular shift in the reflection of light beams, Opt. Commun. 182 (2000) 1-10.

[13] M.A. Ordal, R.J. Bell, R.W. Alexander Jr., R.L. Long, M.R. Querry, Optical properties of $\mathrm{Au}, \mathrm{Ni}$, and $\mathrm{Pb}$ at submillimeter wavelength, Appl. Opt. 26 (1987) 744-752.

[14] E.D. Palik (Ed.), Handbook of Optical Constants of Solids, Academic, New York, 1985. 